University of New Orleans

ScholarWorks@UNO

$1-15-1983$

\title{
Complex reflection coefficients for the parallel and perpendicular polarizations of a film-substrate system
}

\author{
R. M.A. Azzam \\ University of New Orleans, razzam@uno.edu \\ M. Emdadur Rahman Khan
}

Follow this and additional works at: https://scholarworks.uno.edu/ee_facpubs

Part of the Electrical and Electronics Commons, and the Optics Commons

\section{Recommended Citation}

R. M. A. Azzam and M. Emdadur Rahman Khan, "Complex reflection coefficients for the parallel and perpendicular polarizations of a film-substrate system," Appl. Opt. 22, 253-264 (1983)

This Article is brought to you for free and open access by the Department of Electrical Engineering at ScholarWorks@UNO. It has been accepted for inclusion in Electrical Engineering Faculty Publications by an authorized administrator of ScholarWorks@UNO. For more information, please contact scholarworks@uno.edu. 


\title{
Complex reflection coefficients for the parallel and perpendicular polarizations of a film-substrate system
}

\author{
R. M. A. Azzam and M. Emdadur Rahman Khan
}

\begin{abstract}
The complex reflection coefficients $R_{\nu}(\phi, \zeta)$ of a film-substrate system for the parallel $(\nu=p)$ and perpendicular $(\nu=s)$ polarizations are examined in detail as functions of the angle of incidence $\phi\left(0 \leq \phi \leq 90^{\circ}\right)$ and the reduced normalized film thickness $\zeta(0 \leq \zeta<1)$. For definiteness, the reflection of light of wavelength $\lambda=$ $0.6328 \mu \mathrm{m}$ by the air- $\mathrm{SiO}_{2}-\mathrm{Si}$ system is assumed. Families of circles that represent the constant-angle-ofincidence contours, their envelopes, and the associated constant-thickness contours of $R_{p}$ and $R_{s}$ are all presented in the complex plane. Furthermore, the amplitude-reflectance and phase-shift functions, $\left|R_{\nu}\right|(\phi, \zeta)$ and $\arg R_{\nu}(\phi, \zeta)$, are plotted vs $\zeta$ with $\phi$ constant and vs $\phi$ with $\zeta$ constant. It is shown that $R_{p}$ or $R_{s}$ can assume the same complex value at two different angles of incidence (i.e., the film-substrate system can have identical reflection characteristics for a given polarization at two angles) for certain ranges of film thickness. The distinct case of internal reflection is represented by a separate example.
\end{abstract}

\section{Introduction}

The complex amplitude-reflection coefficients $R_{p}$ and $R_{s}$ for light polarized parallel $p$ and perpendicular $s$ to the plane of incidence, reflected from an optically isotropic film-substrate system, are studied as functions of the angle of incidence $\phi$ and film thickness $d$. The ratio of these coefficients $\rho=R_{p} / R_{s}$ (the so-called ellipsometric function) has been subjected to detailed analysis before, ${ }^{1,2}$ and an entire book ${ }^{3}$ is devoted to it for the $\mathrm{SiO}_{2}-\mathrm{Si}$ film-substrate system at different wavelengths. By comparison, the individual reflection coefficients have received only limited attention previously (see, e.g., Refs. 4-6).

The film-substrate system under consideration is shown in Fig. 1. Media 0, 1, and 2 represent the ambient, film, and substrate, respectively, and are assumed to be all homogeneous and optically isotropic. The film has parallel plane boundaries and is of thickness $d$. The ambient and substrate are considered to be of semiinfinite extent. A plane wave of light that is linearly polarized parallel $p$ or perpendicular $s$ to the plane of incidence is incident at an angle $\phi$. The associated

The authors are with University of New Orleans, Electrical Engineering Department, Lakefront, New Orleans, Louisiana 70148.

Received 27 August 1982.

0003-6935/83/020253-00\$01.00/0.

(c) 1983 Optical Society of America. complex amplitude-reflection coefficients are given by ${ }^{7}$ [assuming the $\exp (\mathrm{j} \omega t)$ time dependence and the Nebraska (Muller) conventions]

$$
\begin{aligned}
& R_{\nu}=\frac{r_{01 \nu}+r_{12 \nu} X}{1+r_{01 \nu} r_{12 \nu} X}, \nu=p, s, \\
& X=\exp (-j 2 \pi \zeta) .
\end{aligned}
$$

$r_{i j \nu}$ is Fresnel's complex reflection coefficient of the $i j$ interface for the $\nu$ polarization, and

$$
\zeta=d / D_{\phi}
$$

is the normalized film thickness, where

$$
D_{\phi}=\frac{\lambda}{2}\left(N_{1}^{2}-N_{0}^{2} \sin ^{2} \phi\right)^{-1 / 2}
$$

is the angle-of-incidence-dependent film-thickness period of the periodic reflection coefficients as functions of $d$. $\lambda$ is the free-space wavelength of light, $N_{0}$ and $N_{1}$ are the real refractive indices of the ambient and film media which are presumed to be nonabsorbing. $r_{12 \nu}$, the reflection coefficient of the film-substrate interface, is evaluated at an angle of incidence in the film $\phi^{\prime}$, which is related to $\phi$ by Snell's law:

$$
N_{1} \sin \phi^{\prime}=N_{0} \sin \phi .
$$

Because $r_{i j \nu}$ is function of $\phi$, the film-substrate reflection coefficients $R_{p}$ and $R_{s}$ are functions of $\phi$ and $\zeta$ :

$$
R_{\nu}=R_{\nu}(\phi, \zeta), \quad \nu=p, s .
$$

The arguments $\phi$ and $\zeta$ are limited to the ranges 


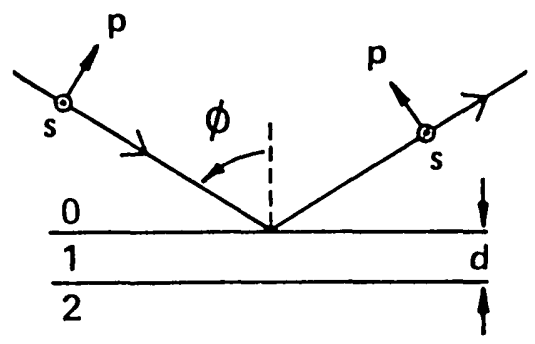

Fig. 1. Reflection of $p$ - or $s$-polarized light at an angle $\phi$ by a film (1)-substrate (2) system. Medium 0 is the ambient, and $d$ is the film thickness.

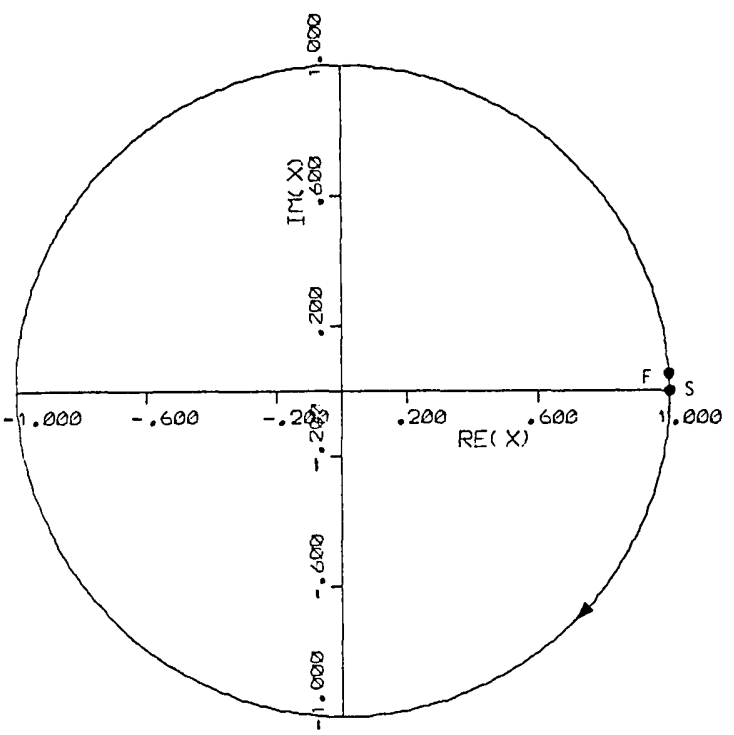

Fig. 2. Unit circle of $X$.

$$
\begin{aligned}
& 0 \leq \phi \leq 90^{\circ}, \\
& 0 \leq \zeta<1 .
\end{aligned}
$$

For transparent films (as we assume) $R_{\nu}$ is a periodic function of $\zeta$ with period 1:

$$
R_{\nu}(\phi, \zeta)=R_{\nu}(\phi, \zeta+m),
$$

where $m$ is any integer. The range given by Eq. (7b) is called the reduced thickness range.

In the following we will consider (and plot) the complex-plane trajectories of $R_{\nu}$ as one of its arguments is held fixed and the other is allowed to vary. The family of curves obtained by holding $\phi$ constant to set values is called the constant-angle-of-incidence contours (CAIC), while those obtained by holding $\zeta$ constant are called the constant-thickness contours (CTC).

Besides examining the behavior of the reflection coefficients in the complex plane, we also consider the associated physically significant real functions

$$
\begin{aligned}
\left|R_{\nu}\right| & =\left|R_{\nu}\right|(\phi, \zeta), \\
\delta_{\nu} & =\arg R_{\nu}(\phi, \zeta), \quad \nu=p, s
\end{aligned}
$$

that represent the amplitude reflectance and reflection phase shift for the $\nu$ polarization, respectively. Families of curves are plotted that illustrate the variation of these real quantities as functions of each argument with the other held constant.

For definiteness, we take the important $\mathrm{SiO}_{2}-\mathrm{Si}$ film-substrate system at the $\mathrm{He}-\mathrm{Ne}$ laser wavelength $\lambda=0.6328 \mu \mathrm{m}$ as an example and assume that the ambient is air or vacuum. We have repeated this work for the $\mathrm{Al}_{2} \mathrm{O}_{3}-\mathrm{Al}$ and $\mathrm{MgF}_{2}$-glass systems at the same wavelength to represent the cases of transparent films on metallic and dielectric substrates, respectively; however, these results are not included here.

In a separate section (Sec. III) we consider the distinctly different situation of internal reflection by a $\mathrm{Ge}-\mathrm{ThF}_{4}$-air system at the $\mathrm{CO}_{2}$-laser wavelength $\lambda=$ $10.6 \mu \mathrm{m}$ as an example.

An interesting conclusion from this investigation is that a film-substrate system can have the same complex reflection coefficient for either the $p$ or $s$ polarization at two different angles of incidence. As discussed in Sec. IV, this is possible only for certain ranges of film thickness.

\section{External Reflection by the Air- $\mathrm{SiO}_{2}-\mathrm{Si}$ System at $\lambda=0.6328 \mu \mathrm{m}$}

An air or vacuum ambient is assumed, $N_{0}=1$, and the refractive indices of $\mathrm{SiO}_{2}$ and $\mathrm{Si}$ are taken to $\mathrm{be}^{3} N_{1}=$ 1.46 and $N_{2}=3.85-j 0.02$, respectively, at $\lambda=0.6328$ $\mu \mathrm{m}$. In the following the parallel and perpendicular polarizations are treated separately.

\section{A. Parallel Polarization}

For a transparent film, the CAICs can be readily proved to be a family of circles. If we return to Eq. (1), we note that $R_{\nu}$ is related to $X$ by a bilinear transformation $^{8}$ whose coefficients are determined by $r_{i j \nu}$. When $\phi$ is assigned a fixed value, $r_{i j \nu}$ become constants. As $\zeta$ is varied from 0 to $1, X$ of Eq. (2) traces the unit circle in the complex plane [Fig. 2] in a clockwise sense beginning at point $S$ and ending at point $F$. ( $S$ and $F$ coincide with $X=1$ but are shown separated for clarity.) Because a bilinear transformation maps a circle onto a circle, ${ }^{8}$ the CAIC is a circle, as first pointed out by Winterbottom. ${ }^{4}$ Figure 3 shows a family of such CAICs that correspond to $\phi=0,10, \ldots, 80^{\circ}$ and $\phi=84$, 86 , and $88^{\circ}$. The CAIC for $\phi=90^{\circ}$ is a null (zero-radius) circle coincident with the point $R_{p}=-1$. The clockwise direction in which all CAICs are traced, as $\zeta$ is increased from 0 to 1 , is indicated by an arrow on the $\phi=60^{\circ}$ contour. Figure 3 shows that the radius of the $\phi=$ constant circle decreases monotonically to zero as $\phi$ is increased from 0 to $90^{\circ}$ with an accompanying leftward shift of the circle center on the real axis to -1 .

There are two interesting member circles of the family of CAICs that are not shown in Fig. 3. One corresponds to the Brewster angle of the ambient-film interface,

$$
\phi=\phi_{B 01}=\tan ^{-1} 1.46=55.59^{\circ},
$$

which is a circle centered on the origin, so that $\left|R_{p}\right|$ is a constant independent of $\zeta$. This is represented by the 


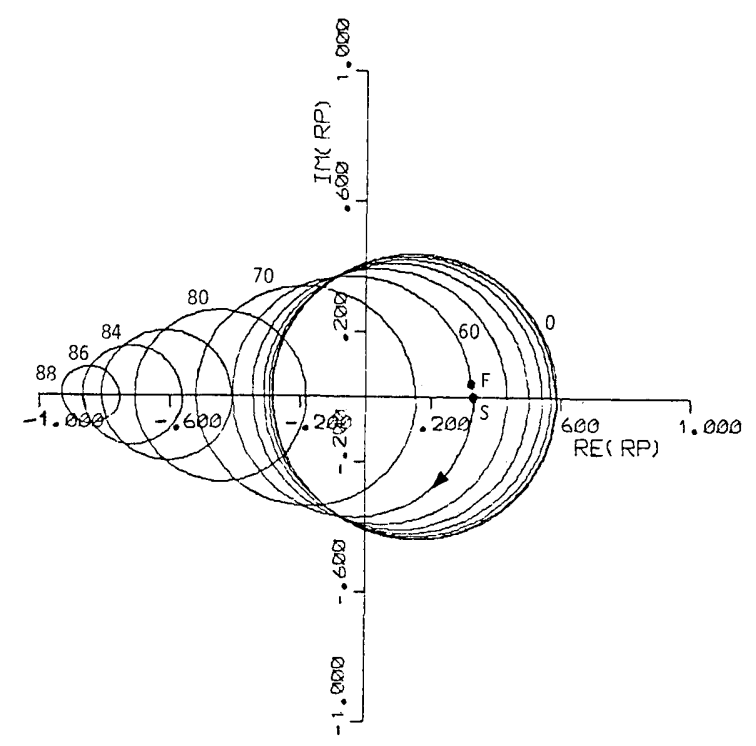

Fig. 3. CAICs of $R_{p}$ as a family of circles with the angle of incidence $\phi$ marked on each contour. The air- $\mathrm{SiO}_{2}-\mathrm{Si}$ system is assumed at $\lambda=0.6328 \mu \mathrm{m}$.

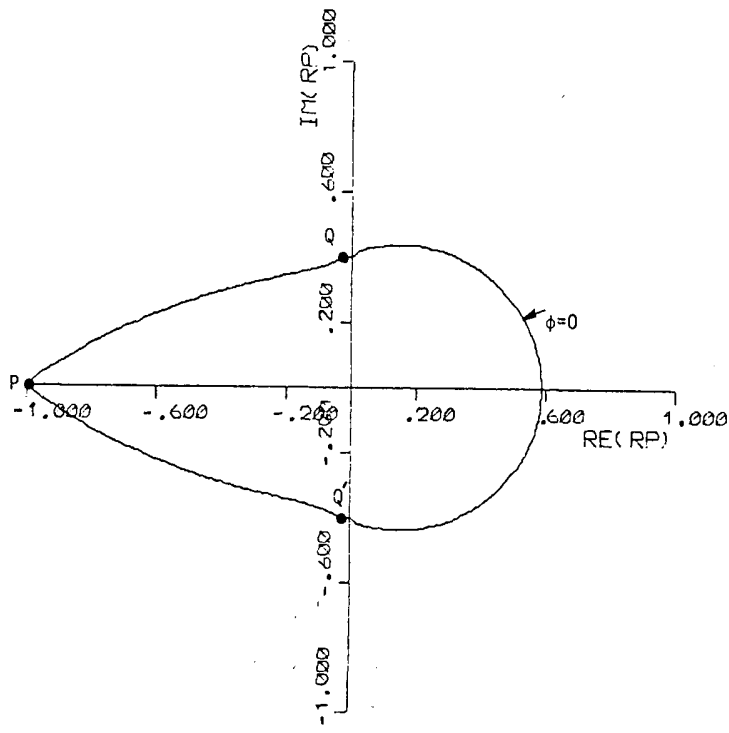

Fig. 4. Envelope curves $P Q$ and $P Q^{\prime}$ of the CAICs of Fig. 3 and the circle arc $Q Q^{\prime}$ of the $\phi=0$ circle.

horizontal dashed line in Fig. 8(a) and is referred to as the Abelès condition. ${ }^{9}$ The second circle passes through the origin, so that $R_{p}=0$ at a certain $\phi=\phi_{p}$ and $\zeta=\zeta_{p}$. From Ref. 1 we find that such polarizing angle and thickness are $\phi_{p}=75.44^{\circ}$ and $\zeta_{p}=0.999$.

Figure 4 shows the envelope ${ }^{10}$ of the CAICs of Fig. 3 as consisting of two branches $P Q$ and $P Q^{\prime}$, where $Q$ and $Q^{\prime}$ are the points where the envelope touches the $\phi=0$ CAIC. Also shown in Fig. 4 is the circle arc $\overline{Q Q}^{\prime}$ of the $\phi=0$ CAIC. The closed contour that consists of the envelope curves $P Q$ and $P Q^{\prime}$ and the circle arc $Q^{\prime} Q^{\prime}$ and the area that it encloses define the domain of all possible values of the complex reflection coefficient $R_{p}$ that can be generated by the air- $\mathrm{SiO}_{2}-\mathrm{Si}$ system at $\lambda=0.6328$ $\mu \mathrm{m}$ as the film thickness and angle of incidence are allowed to take all possible values. The area of the complex plane between the closed contour of Fig. 4 and the unit circle represents $R_{p}$ values that cannot be generated by this specific system at this particular wavelength.

Now we turn our attention to the CTCs. Figure 5 shows the film thickness period $D_{\phi}$ in micrometers vs $\phi$ in degrees as determined by Eq. (4) with $N_{0}=1, N_{1}$ $=1.46$, and $\lambda=0.6328 \mu \mathrm{m}$. The thickness range $0 \leq d$ $\leq D_{90}=297.4 \mathrm{~nm}$ is divided into two ranges: (1) $0 \leq$ $d \leq D_{0}=216.7 \mathrm{~nm}$ and (2) $D_{0} \leq d \leq D_{90}$. For a given thickness in range (1) $\phi$ is varied from 0 to $90^{\circ}$, while in range (2) $\phi$ is restricted from $\bar{\phi}$ to $90^{\circ}$, where $\bar{\phi}$ corresponds to the point of intersection of the $d=$ constant line with the $D_{\phi}$ vs $\phi$ curve and is given from Eq. (4) by

$$
\bar{\phi}=\arcsin \left[\left(N_{1} / N_{0}\right)^{2}-\left(\lambda / 2 d N_{0}\right)^{2}\right]^{1 / 2} .
$$

Limiting $\phi$ to the range $\bar{\phi} \leq \phi \leq 90^{\circ}$, when $D_{0} \leq d \leq$ $D_{90}$, keeps $\zeta$ within the reduced thickness range [Eq. (7b)] and avoids repetition of $R_{p}$ due to its periodicity as a function of $\zeta$.

Figure 6 shows a family of CTCs that correspond to $d=(m / 20) D_{0}$, where $m=0,1,2, \ldots, 20$. The direction in which a CTC is traced, as $\phi$ increases from 0 to $90^{\circ}$, is represented by the arrow on the contour for $m=1$. The locus of the starting point $S$ is, of course, the $\phi=$ 0 CAIC. The final point $F$ corresponds to $R_{p}=-1$ at $\phi=90^{\circ}$.

Figure 7 shows CTCs that correspond to $d=D_{0}+$ $(m / 10)\left(D_{90}-D_{0}\right)$, where $m=0,1,2, \ldots, 10$. The $m=$ $10, d=D_{90}$, CTC is a null contour that coincides with point $F$, where $R_{p_{-}}=-1$. Along each CTC of this group $\phi$ increases from $\bar{\phi}$ [Eq. (12)] to $90^{\circ}$. The $m=9$ CTC

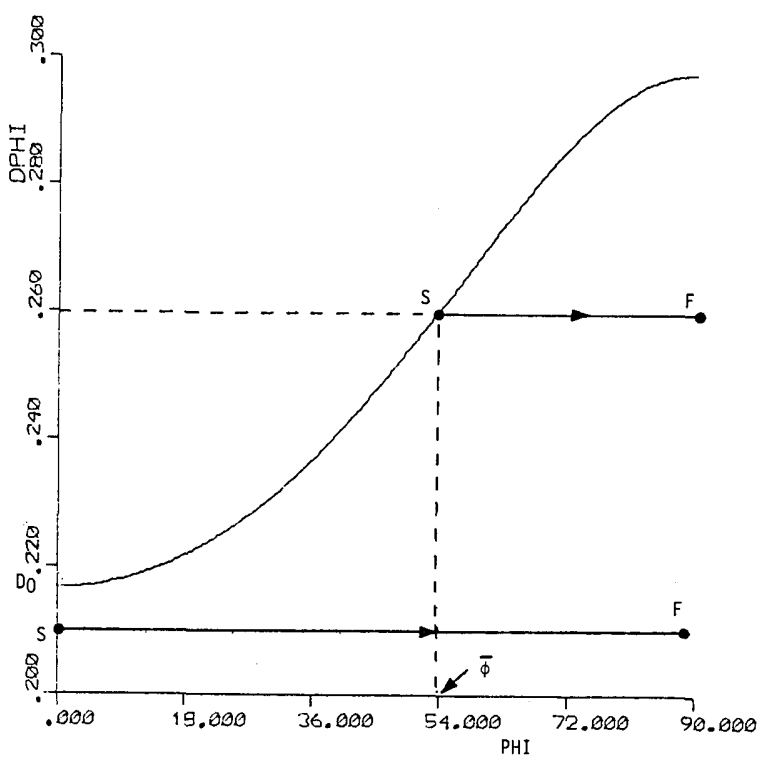

Fig. 5. Film thickness period $D_{\phi}$ vs angle of incidence $\phi$ for an air ambient and $\mathrm{SiO}_{2}$ film at $\lambda=0.6328 \mu \mathrm{m}$. 


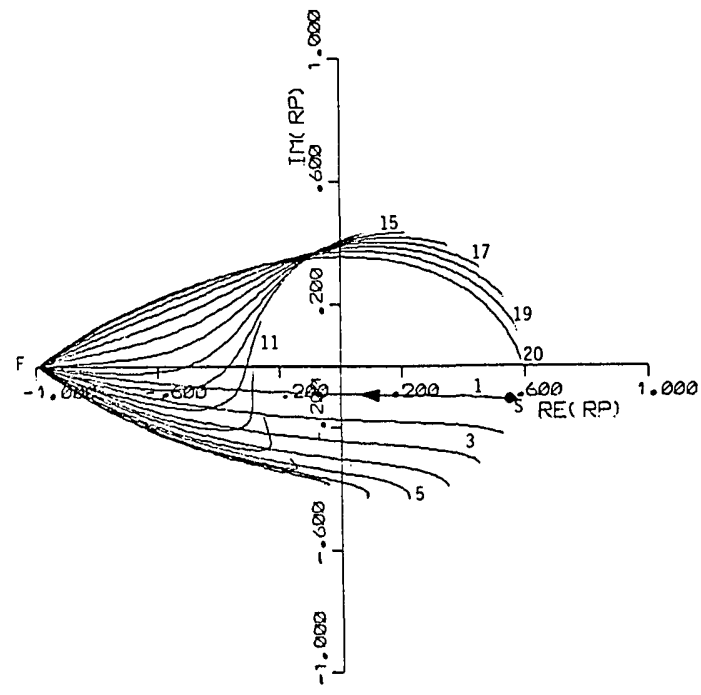

Fig. 6. CTCs of $R_{p}$ for film thicknesses $d=(m / 20) D_{0}, m=0,1,2$, $\ldots, 20$. The air $-\mathrm{SiO}_{2}-\mathrm{Si}$ system is assumed at $\lambda=0.6328 \mu \mathrm{m}$. Along each CTC, $\phi$ varies from 0 to $90^{\circ}$.

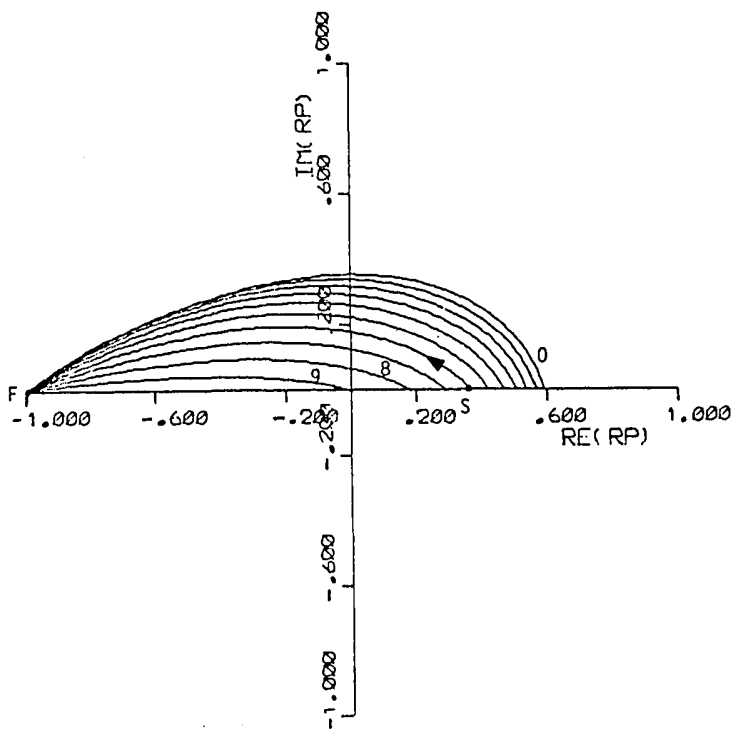

Fig. 7. Same as Fig. 6 for thicknesses $d=D_{0}+(m / 10)\left(D_{90}-\mathrm{D}_{0}\right)$, $m=0,1,2, \ldots, 10$. Along each CTC, $\phi$ varies from $\bar{\phi}$, Eq. (12), to $90^{\circ}$.

appears to begin at the origin, and the associated thickness $(289.36 \mathrm{~nm})$ is indeed very nearly that which is required to suppress the $p$ polarization ${ }^{1}(d=289.17$ $\mathrm{nm}$ ).

Figure 8(a) shows how the amplitude reflectance $\left|R_{p}\right|$ varies with the normalized film thickness $\zeta$ along each CAIC of Fig. 3. The maxima and minima of reflectance appear to be located at $\zeta=0,1$ and $\zeta=0.5$. Such locations are exact only for a transparent film on a transparent substrate, but they very nearly apply to the $\mathrm{SiO}_{2}-\mathrm{Si}$ system because of the numerically small $(0.02)$ extinction coefficient of $\mathrm{Si}$ at $\lambda=0.6328 \mu \mathrm{m}$. At grazing incidence, $\phi=90^{\circ},\left|R_{p}\right|$ is constant equal to 1 independent of $\zeta .\left|R_{p}\right|$ is also constant for all $\zeta$ when $\phi=$
$\phi_{B 01}=55.59^{\circ}$, the Brewster angle of the air- $\mathrm{SiO}_{2}$ interface (Abelès condition ${ }^{9}$ ). This is represented by the dashed line in Fig. 8(a). The minimum of $\left|R_{p}\right|$ reaches zero at the polarizing angle ${ }^{1} \phi=\phi_{p}=75.44^{\circ}$.

The reflection phase shift, $\delta_{p}=\arg R_{p}$, is plotted in Fig. 8(b) as a function of $\zeta$ for each CAIC of Fig. 3. Note that for $\phi<\phi_{p}=75.44^{\circ}$ the CAIC encloses the origin, and $\delta_{p}$ monotonically decreases with $\zeta$ from $360^{\circ}$ to 0 over the range $0<\zeta<1$. For $\phi>\phi_{p}$, the CAIC lies entirely in the left half plane, and $\delta_{p}$ is limited to a range around $180^{\circ}$ and goes through a maximum and a minimum in that range. That $\delta_{p}=180^{\circ}$ at $\zeta=0.5$ independent of $\phi$, as appears in Fig. 8(b), is strictly true for

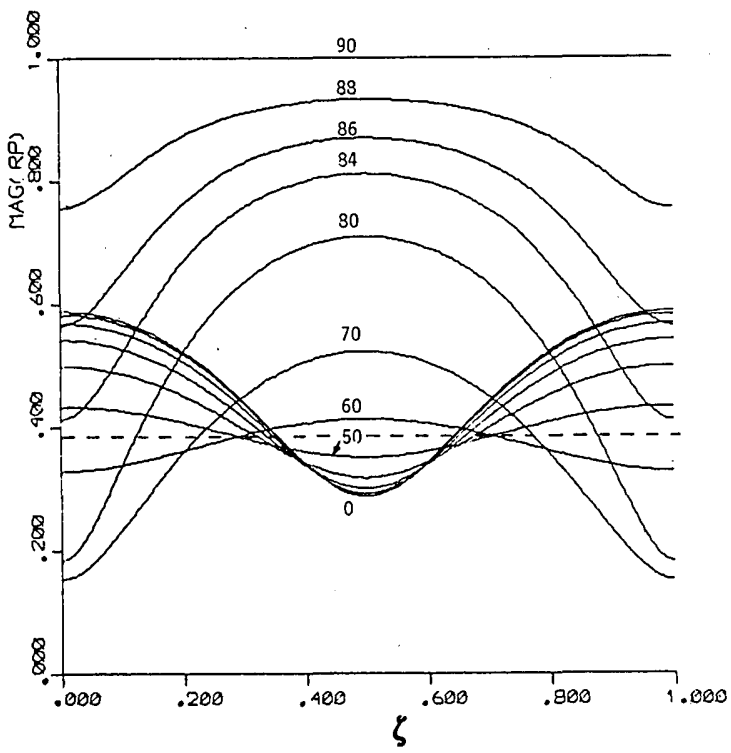

(a)

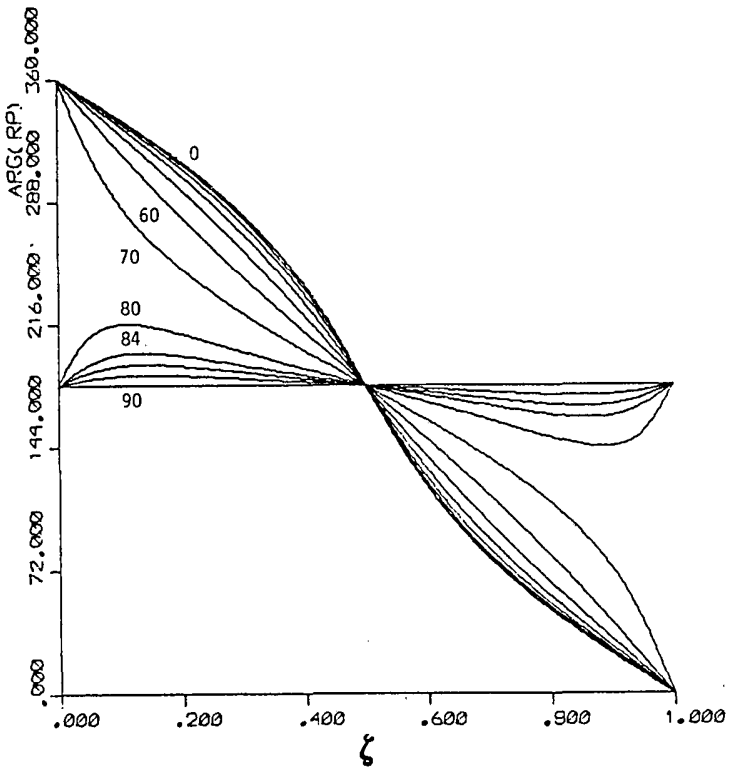

(b)

Fig. 8. Amplitude reflectance $\left|R_{p}\right|$ (a) and reflection phase shift $\delta_{p}$ (b) vs normalized film thickness $\zeta$ with the angle of incidence $\phi$ as a parameter marked by each curve for the air $-\mathrm{SiO}_{2}-\mathrm{Si}$ system at $\lambda=$ $0.6328 \mu \mathrm{m}$. 


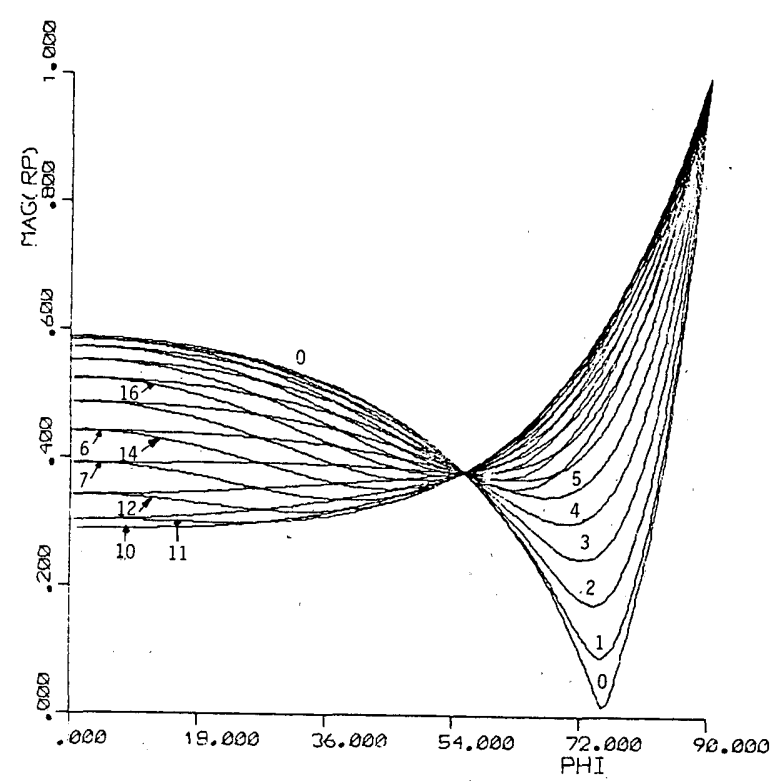

(a)

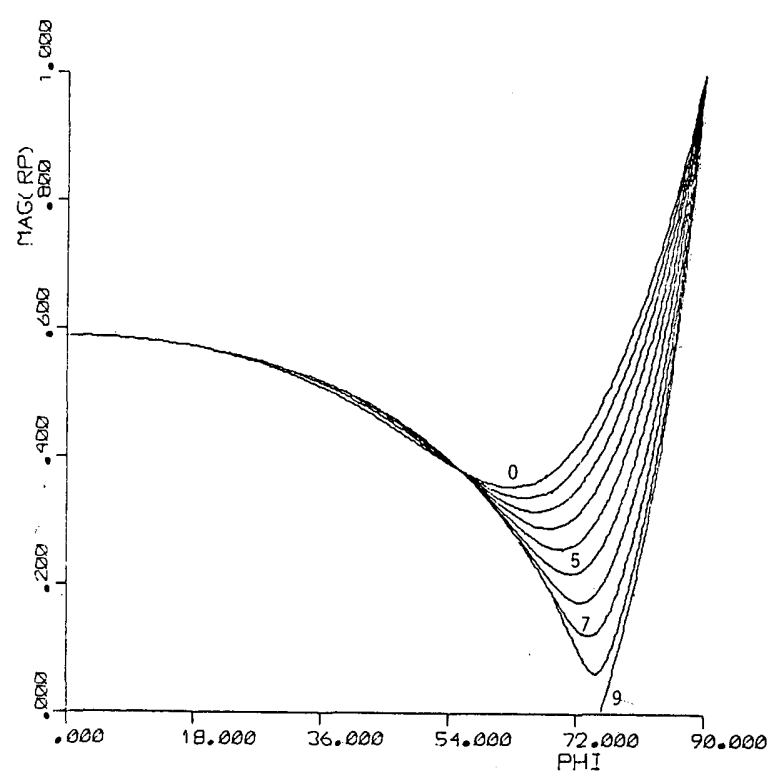

(b)

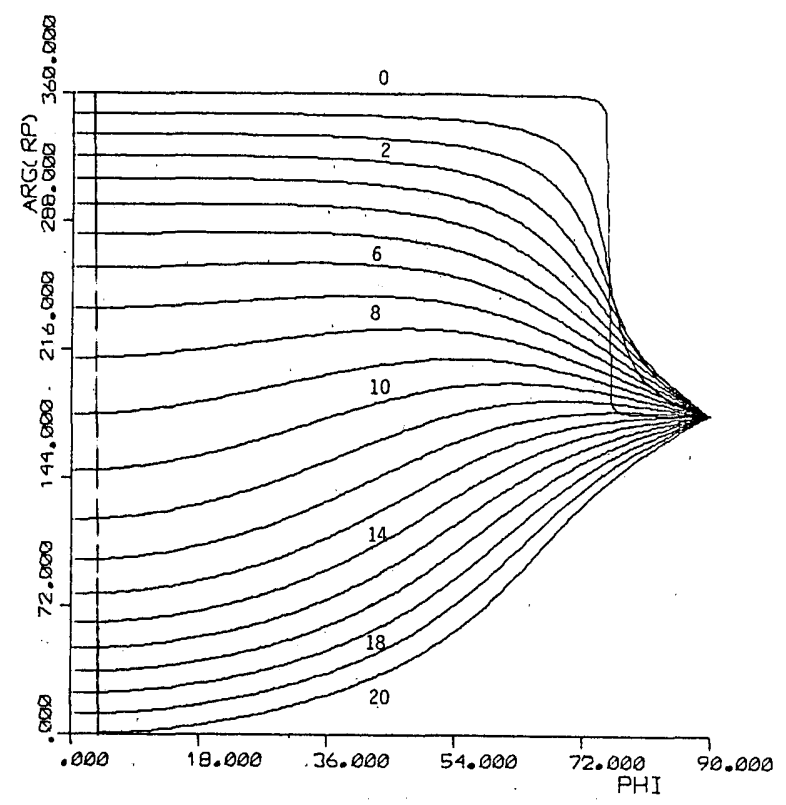

(c)

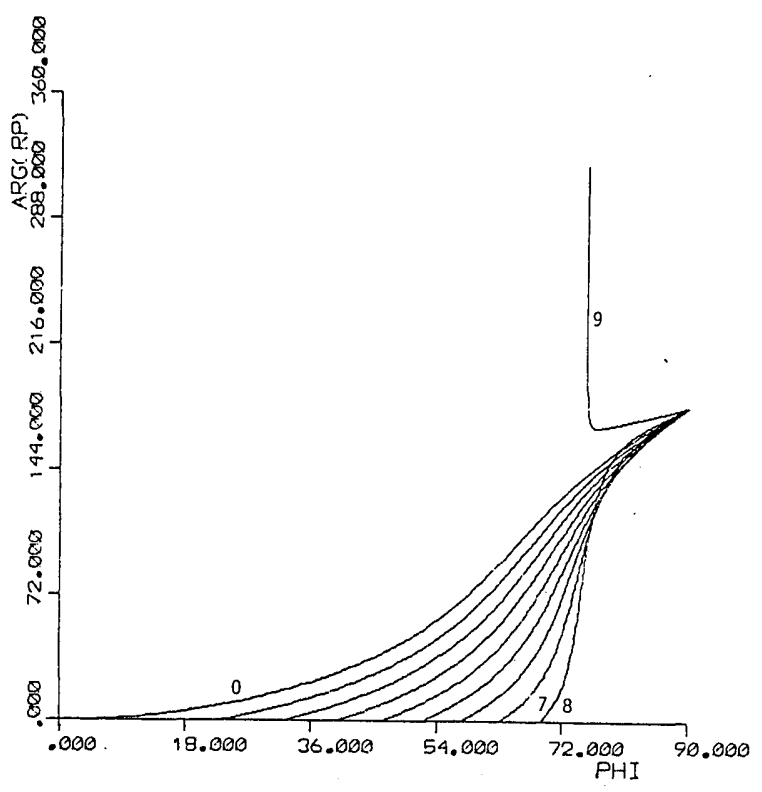

(d)

Fig. 9. Amplitude reflectance $\left|R_{p}\right|$ (a) and reflection phase shift $\delta_{p}$ (c) vs angle of incidence $\phi$ along the CTCs of Fig. 6 . (b) and (d) depict the same quantities for the CTCs of Fig. 7.

a transparent film on a transparent substrate and is very nearly so for the present $\mathrm{SiO}_{2}-\mathrm{Si}$ system.

Figures 9(a) and (b) show $\left|R_{p}\right|$ vs $\phi$ along each CTC of Figs. 6 and 7, respectively. A noteworthy feature of these figures is the prominent display of the Abelès condition by the passage of all curves through one common point at $\phi=55.59^{\circ}$.

Also interesting to note in Fig. 9 is the shift of the pseudo-Brewster angle $\phi_{\mathrm{pB}}$ of minimum parallel reflectance as a function of film thickness $d$. As $m$ and $d$ increase from $0, \phi_{\mathrm{pB}}$ decreases monotonically from $\phi_{\mathrm{pB}}$ $=75.44^{\circ}$ of bare Si to 0 when $m \approx 8$ and $d \approx 86.7 \mathrm{~nm}$. At $\phi_{B 01}=55.59^{\circ}$ the minimum parallel reflectance reaches its maximum possible value, which is equal to that of bare Si at that angle $(=0.381)$. A further in- crease of $m$ from $\approx 8$ to $\approx 10$ (and of $d$ from $\approx 86.7$ to $\approx 108.4 \mathrm{~nm}$ ) leads to an $\left|R_{p}\right|$ vs $\phi$ curve that is monotonically increasing without a minimum at oblique incidence. This interval of thickness, over which the Brewster phenomenon no longer exists, appears from Fig. 6 to correspond to the smaller arc of the $\phi=0$ CAIC that complements the arc $\widehat{Q} Q^{\prime}$ of Fig. 4. As $d$ sweeps the interval from $d \approx 108.4 \mathrm{~nm}$ to $d=d_{p}=289.17 \mathrm{~nm}$, $\phi_{\mathrm{pB}}$ reappears and increases from 0 to $\phi_{p}=75.44^{\circ} . d_{p}$ and $\phi_{p}$ are the polarizing film thickness and angle of incidence, respectively.

Figures 9 (c) and (d) show the $\delta_{p}$ vs $\phi$ phase curves for the thickness ranges $0 \leq d \leq D_{0}$ and $D_{0} \leq d \leq D_{90}$, respectively. The same discrete thickness values as in Figs. 9(a) and (b) are assumed, respectively. 


\section{B. Perpendicular Polarization}

The complex reflection coefficient for $s$-polarized light $R_{s}$ of the air- $-\mathrm{SiO}_{2}-\mathrm{Si}$ system at $\lambda=0.6328 \mu \mathrm{m}$ has been examined as a function of the angle of incidence $\phi$ and normalized film thickness $\zeta$ in the same degree of detail as was presented for $p$-polarized light in Sec. II. The same constant values of the parameters $\phi$ and $\zeta$ are selected to facilitate comparison between the behavior of $R_{s}$ and $R_{p}$. The results, which are given in Figs. $10-13$, will be briefly outlined.

Figure 10(a) shows a family of CAICs (circles) of $R_{s}$ at the angles marked by each curve. For $\phi=90^{\circ}$, the CAIC reduces to the single point $R_{s}=-1$. The locus of centers is the real axis, as is the case of the CAICs of $R_{p}$. As $\phi$ increases from 0 to $90^{\circ}$, the center of the CAIC moves leftward to -1 , and its radius initially increases to a maximum $\left(\sim \phi=50^{\circ}\right)$, then drops to zero. There

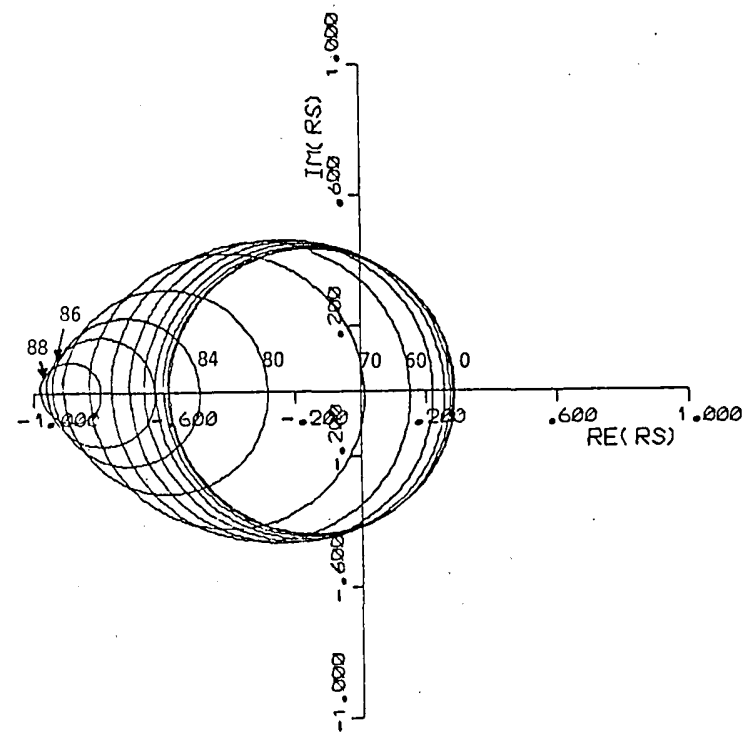

(a)

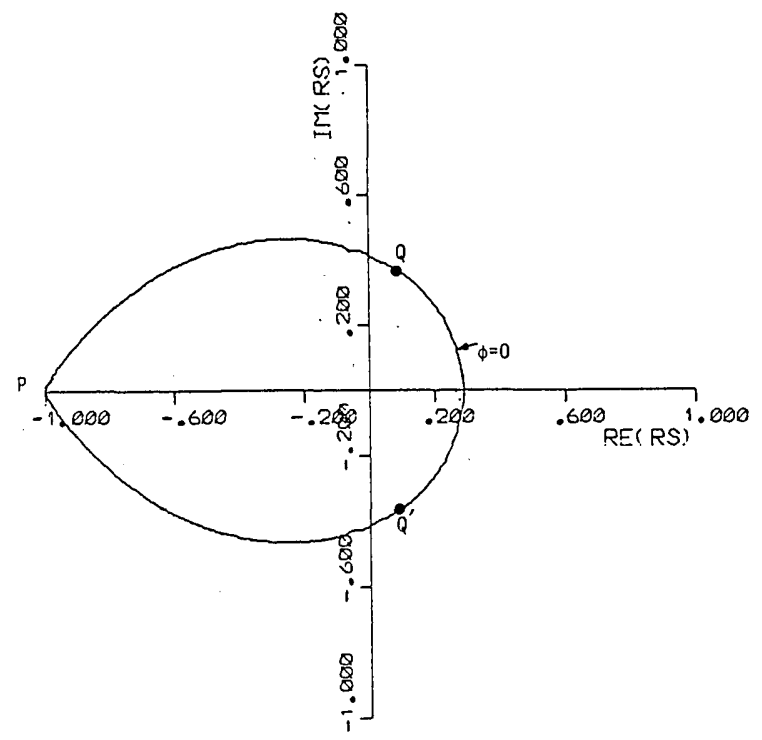

(b)

Fig. 10. CAICs of $R_{s}$ (a) and their envelope (b) for the air- $\mathrm{SiO}_{2}-\mathrm{Si}$ system at $\lambda=0.6328 \mu \mathrm{m}$. is no circle with the origin as center, as the Abelès condition is known to be unique to the $p$ polarization alone. The circle of $\phi=\phi_{s}=70.56^{\circ}$ (not shown) passes through the origin when $d=d_{s}=141.77 \mathrm{~nm}\left(\zeta=\zeta_{s}=\right.$ $0.499)$. $\phi_{s}$ and $d_{s}$ represent the angle of incidence and (the least) film thickness that are required to extinguish $s$-polarized light upon reflection. ${ }^{1}$

Figure 10(b) shows a closed contour that consists of the envelope curves $P Q$ and $P Q^{\prime}$ of the CAICs of Fig. 10 (a) and the circle arc $Q Q^{\prime}$ of the $\phi=0$ CAIC. Points on and inside this closed contour represent all possible values of the complex reflection coefficient $R_{s}$ of the air- $\mathrm{SiO}_{2}-\mathrm{Si}$ system at $\lambda=0.6328 \mu \mathrm{m}$.

Figure 11 shows CTCs of $R_{s}$. The specific thickness values and the associated ranges of $\phi$ are exactly the same as was described in connection with Figs. 5-7.

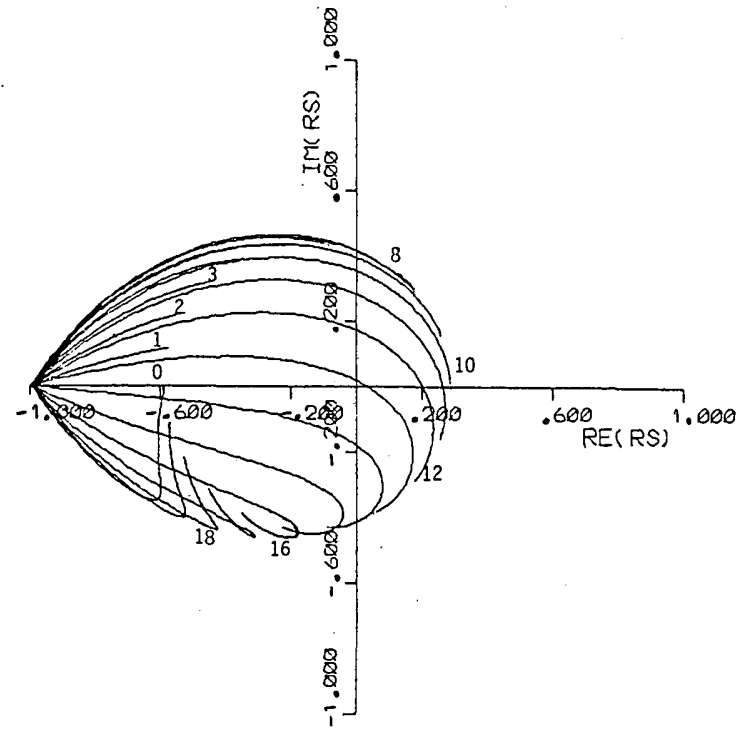

(a)

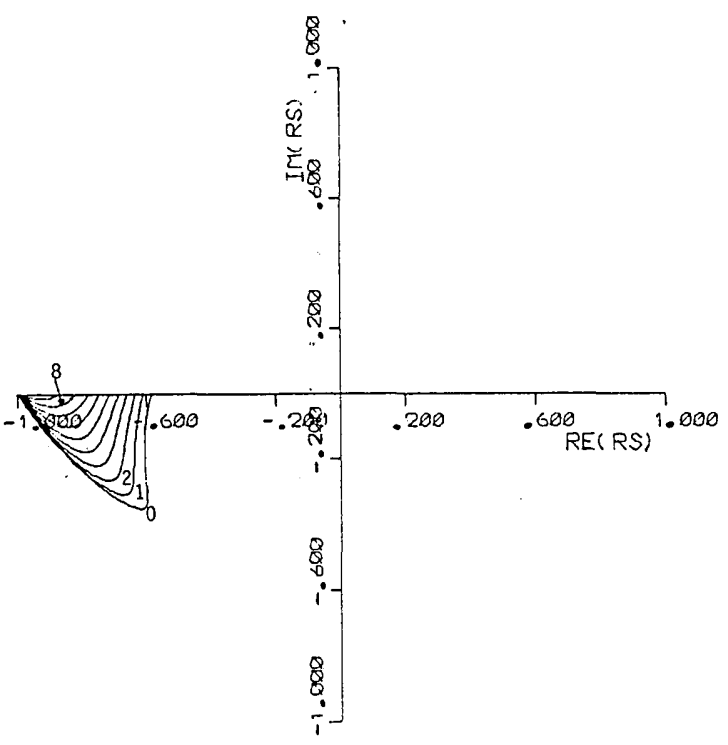

(b)

Fig. 11. CTCs of $R_{s}$ for the air- $\mathrm{SiO}_{2}-\mathrm{Si}$ system at $\lambda=0.6328 \mu \mathrm{m}$. (a) $d=(m / 20) D_{0}, m=0,1,2, \ldots, 20$, and (b) $d=D_{0}+(m / 10)\left(D_{90}-D_{0}\right)$, $m=0,1,2, \ldots, 10$. 
Figures 12(a) and (b) give $\left|R_{s}\right|$ vs $\zeta$ and $\delta_{s}\left(=\arg R_{s}\right)$ vs $\zeta$, respectively, along each and every CAIC of Fig. 10(a). In Fig. 12(a) the maxima and minima of $\left|R_{s}\right|$ appear to occur at $\zeta=0,1$ and $\zeta=0.5$ because of the numerically small extinction coefficient of $\mathrm{Si}$ as was true with $\left|R_{p}\right|$. Similar remarks apply to the values $(0, \pi$, or $2 \pi$ ) of $\delta_{s}$ [Fig. 12(b)] at $\zeta=0,0.5,1$.

Figures 13(a) and (b) give $\left|R_{s}\right|$ vs $\phi$ along the CTCs of Figs. 11(a) and (b), respectively, while Figs. 13(c) and (d) give the corresponding $\delta_{s}$ vs $\phi$ curves. In Fig. 13(a) it is interesting to note that a minimum of $\left|R_{s}\right|$ at

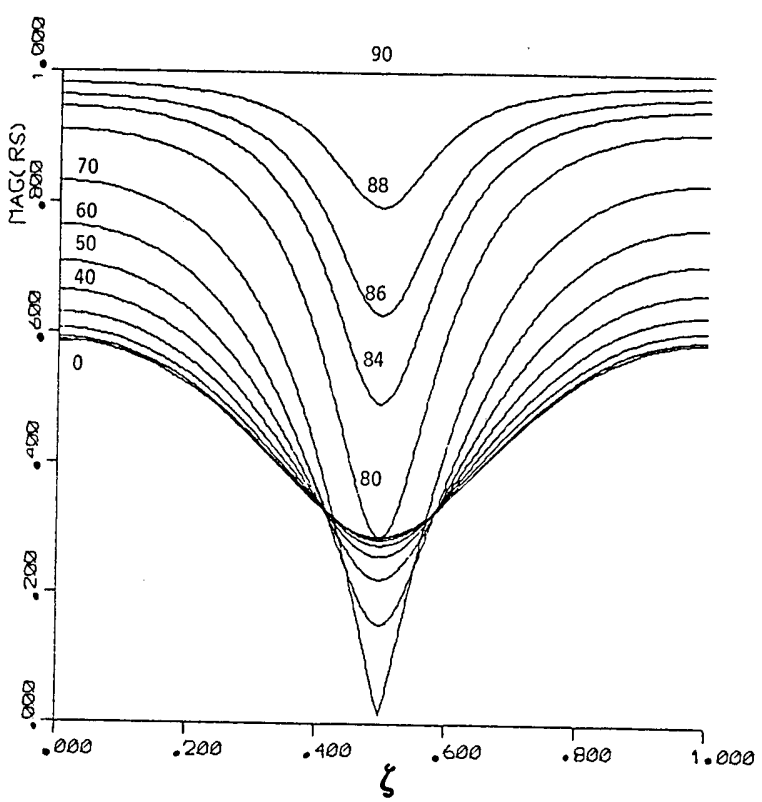

(a)

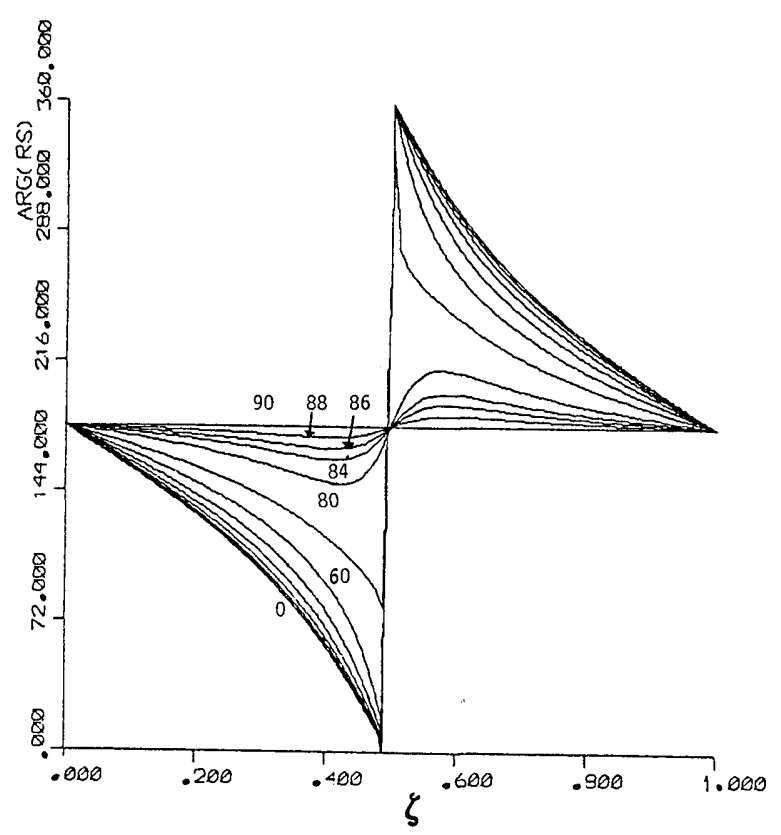

(b)

Fig. 12. Amplitude reflectance $\left|R_{s}\right|$ (a) and reflection phase shift $\delta_{s}$ (b) vs normalized film thickness $\zeta$ with the angle of incidence $\phi$ as a parameter marked by each curve for the air-SiO ${ }_{2}-\mathrm{Si}$ system at $\lambda=$ $0.6328 \mu \mathrm{m}$. oblique incidence occurs only for a limited interval of the reduced thickness around $d_{s}=141.77 \mathrm{~nm}(10 \lesssim m$ $\lesssim 17,108 \mathrm{~nm} \lesssim d \lesssim 184 \mathrm{~nm})$.

\section{Internal Reflection by the $\mathrm{Ge}^{-} \mathrm{ThF}_{4}$-Air System at $\lambda=\mathbf{1 0 . 6} \mu \mathrm{m}$}

We consider as an example the complex coefficients of internal reflection $R_{p}$ and $R_{s}$ of $p$ - and $s$-polarized 10.6- $\mu \mathrm{m}$ radiation by the $\mathrm{Ge}-\mathrm{ThF}_{4}$-air system. The refractive indices of $\mathrm{Ge}, \mathrm{ThF}_{4}$, and air are taken to be $^{11,12} N_{0}=4, N_{1}=1.37$, and $N_{2}=1$, respectively. We discuss separately the cases of partial and total reflection at angles of incidence $\phi$ below and above the critical angle $\phi_{c}$ of the $\mathrm{Ge}$-air interface:

$$
\phi_{c}=\arcsin \left(N_{2} / N_{0}\right)=\arcsin (0.25)=14.48^{\circ} .
$$

For brevity we limit ourselves to the characteristic CAICs and CTCs.

\section{A. Partial Reflection at $\phi<\phi_{c}$}

Figure 14 shows CAICs of $R_{p}$ at selected angles $\phi<$ $\phi_{c}=14.48^{\circ}$. As $\phi$ is increased from 0 to a certain angle $\phi_{0}$, the radius of the $\phi=$ constant circle shrinks monotonically from that of the $\phi=0$ circle to 0 . This is represented by a distinct family of circles that lie within the $\phi=0$ circle. $\phi_{0}$ that leads to a null circle of $R_{p}$ is determined by

$$
\csc ^{2} \phi_{0}=\left(N_{0} / N_{2}\right)^{2}+\left(N_{0} / N_{1}\right)^{2},
$$

which is equal to $11.65^{\circ}$ for the $\mathrm{Ge}-\mathrm{ThF}_{4}$-air system under consideration. Incidence at $\phi=\phi_{0}$ leads to incidence of light on the film-substrate $\left(\mathrm{ThF}_{4}\right.$-air) interface at its Brewster angle, so that the net reflection coefficient $R_{p}$ becomes independent of film thickness ${ }^{13}$ and is equal to

$$
R_{p}=r_{02 p}=-0.413 .
$$

As $\phi$ increases beyond $\phi_{0}$ and tends to $\phi_{c}$, the circle of $R_{p}$ expands, and its radius increases from 0 and tends to 1 (Fig. 14). At $\phi=\phi_{c}$, the circle collapses to a single point, $R_{p}=1$. It is interesting that all possible values of the complex reflection coefficient $R_{p}$ (represented by all points inside the unit circle of the complex plane) are generated when $\phi$ scans the brief interval $\phi_{0}=$ $11.65^{\circ} \leq \phi<14.48^{\circ}=\phi_{c}$. Scanning the interval $0 \leq \phi$ $\leq \phi_{0}$ repeats the generation of complex values of $R_{p}$ on and within the $\phi=0$ CAIC.

Figure 15 shows a family of CTCs associated with the CAICs of Fig. 14. These contours, which all pass through a common point given by Eq. (15), are generated by allowing the film thickness to assume the values $d=(m / 5) D_{0}$, where $D_{0}=3.869 \mu \mathrm{m}$ and $m=0,1,2, \ldots$, 5 , and varying $\phi$ from 0 to $\phi_{c}$.

Figure 16 shows CAICs (circles) of $R_{s}$ for $\phi<\phi_{c}$. The circles are nonintersecting with radii that increase monotonically as $\phi$ increases from 0 to $\phi_{c}$. The CAIC tends to the unit circle as $\phi \rightarrow \phi_{c}$. However, at $\phi=\phi_{c}$, the CAIC collapses to the single point $R_{s}=1$ as with $R_{p}$. Note that the area inside the $\phi=0$ circle represents complex values of $R_{s}$ that cannot be generated by this system. In contrast with the CAICs of external reflection (by the air- $\mathrm{SiO}_{2}-\mathrm{Si}$ system at $\lambda=0.6328 \mu \mathrm{m}$, 


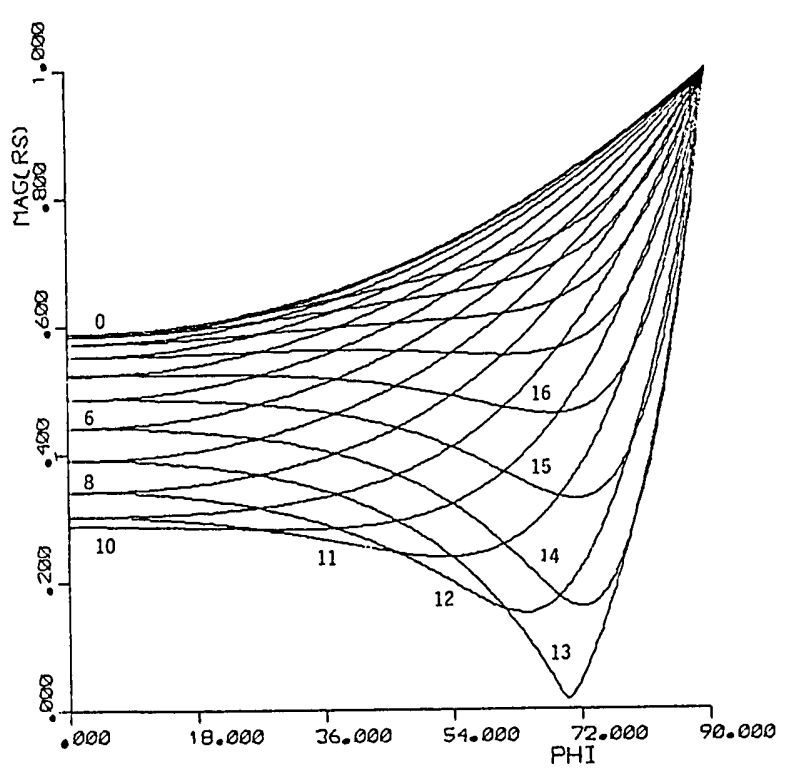

(a)

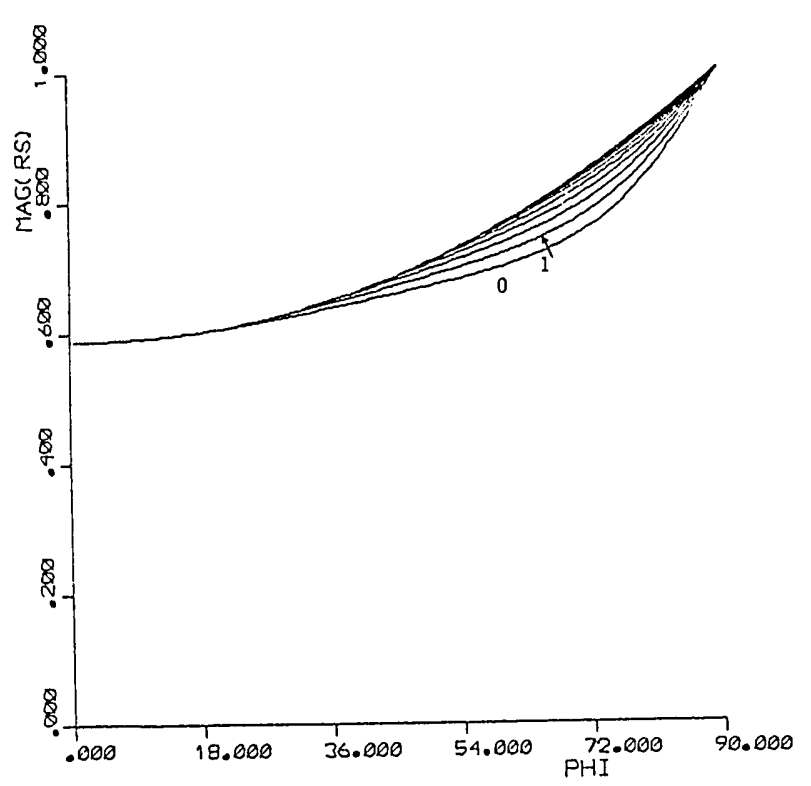

(b)

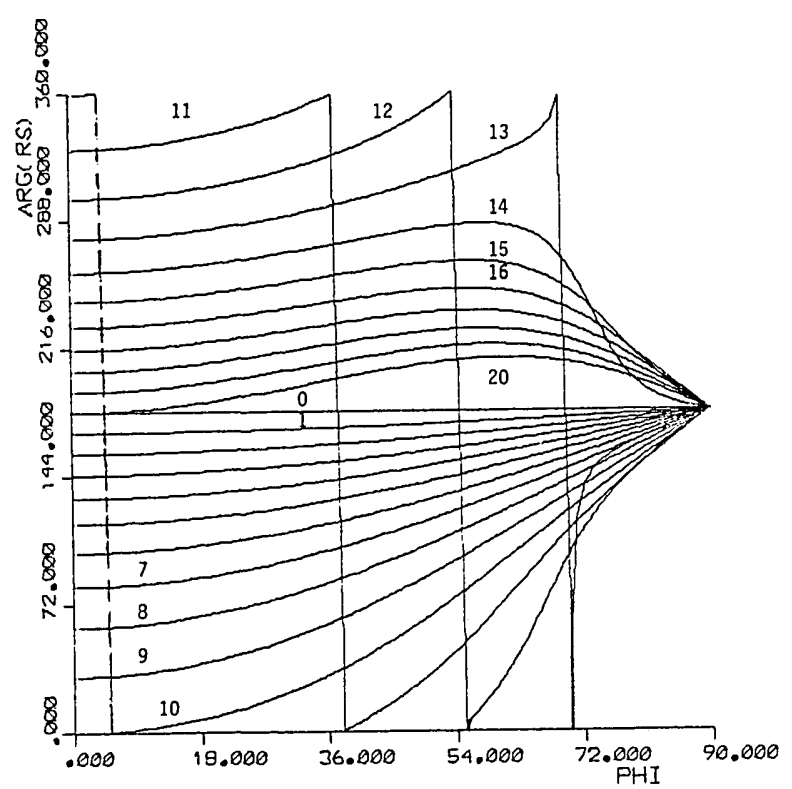

(c)

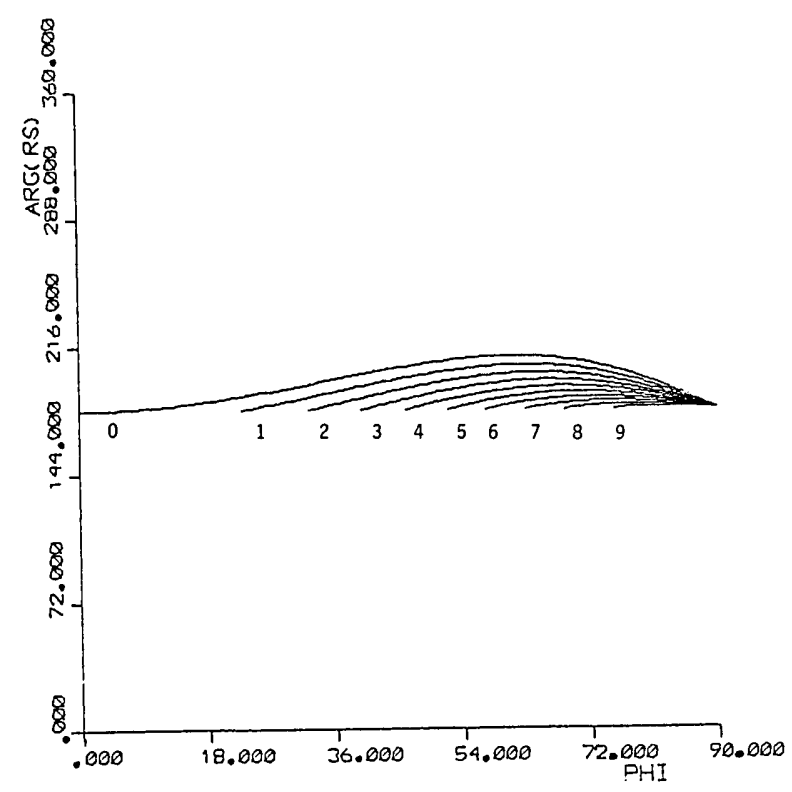

(d)

Fig. 13. Amplitude reflectance $\left|R_{s}\right|$ (a) and reflection phase shift $\delta_{s}$ (c) vs angle of incidence $\phi$ along the CTCs of Fig. 11(a). (b) and (d) depict the same quantities for the CTCs of Fig. 11(b).

Figs. 3 and 5), the families of circles of Figs. 14 and 16 do not have envelopes.

Figure 17 shows the associated family of CTCs of $R_{s}$ that correspond to $d=(m / 5) D_{0}$, where $D_{0}=3.869 \mu \mathrm{m}$ and $m=0,1,2, \ldots, 5$.

\section{B. Total Reflection, $\phi>\phi_{c}$}

When $\phi>\phi_{c}, D_{\phi}$ of Eq. (4) and $\zeta$ of Eq. (3) become purely imaginary. In this case, $X$ of Eq. (2) becomes real, nonperiodic, and traces the segment of the real axis between $X=1$ and $X=0$ as $d$ and $\zeta$ increase from 0 to $\infty$, respectively. At a given $\phi, R_{\nu}(\nu=p$ or $s)$, which is the image of $X$ through a bilinear transformation [Eq. (1)], traces an arc of the unit circle that begins at $R_{02 \nu}$, when $d=\zeta=0$, and ends at $R_{01 \nu}$, when $d=\zeta=\infty$. $R_{02 \nu}$

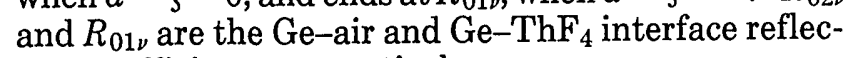
tion coefficients, respectively.

Figures 18(a) and (b) show the CAICs of $R_{p}$ and $R_{s}$, respectively, as overlapping and nonoverlapping circle arcs of the unit circle. The CAIC at $\phi=90^{\circ}$ is a null arc at $R_{\nu}=-1$.

All CTCs also coincide with the unit circle and are not plotted. 


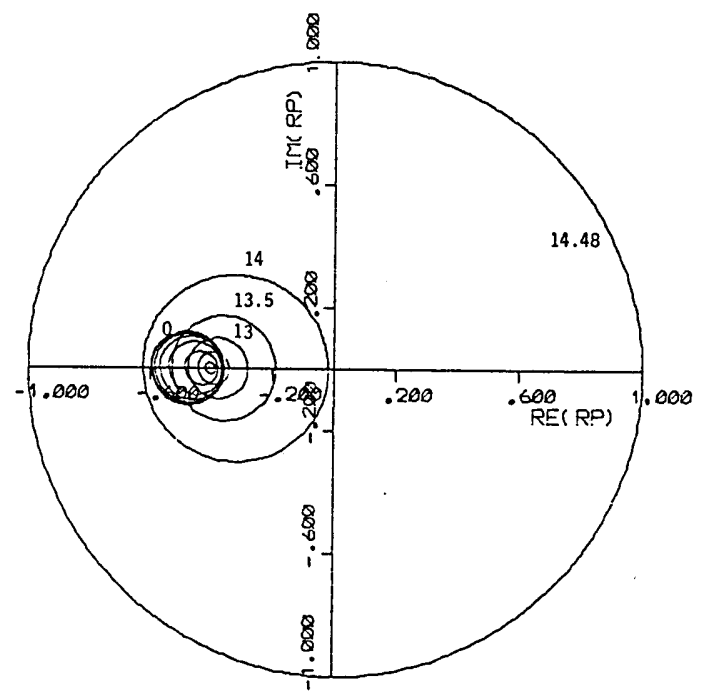

Fig. 14. CAICs of $R_{p}$ for partial internal reflection below the critical angle for the $\mathrm{Ge}_{-} \mathrm{ThF}_{4}$-air system at $\lambda=10.6 \mu \mathrm{m}$. The distinct circles that lie within the $\phi=0$ circle correspond to $\phi=4,6,8,10$, and $12^{\circ}$ in order of decreasing radius.

\section{Can a Film-Substrate System Have Identical Complex $p$ - or $s$-Reflection Coefficients at Two Different Angles of Incidence?}

The answer to the title of this section is yes. This interesting characteristic, which, to our knowledge, has not been recognized before, is evident from Fig. 11(a). Specifically, it follows from the fact that the CTC of $R_{s}$ for $m=17(d=184.21 \mathrm{~nm})$ makes a loop. The point where the CTC intersects itself represents one and the same complex reflection coefficient for the $s$ polarization at two different angles of incidence. Recall that this is the air- $\mathrm{SiO}_{2}-\mathrm{Si}$ system at $\lambda=0.6328 \mu \mathrm{m}$. This property of complex $R_{s}$ repeating itself at two angles of

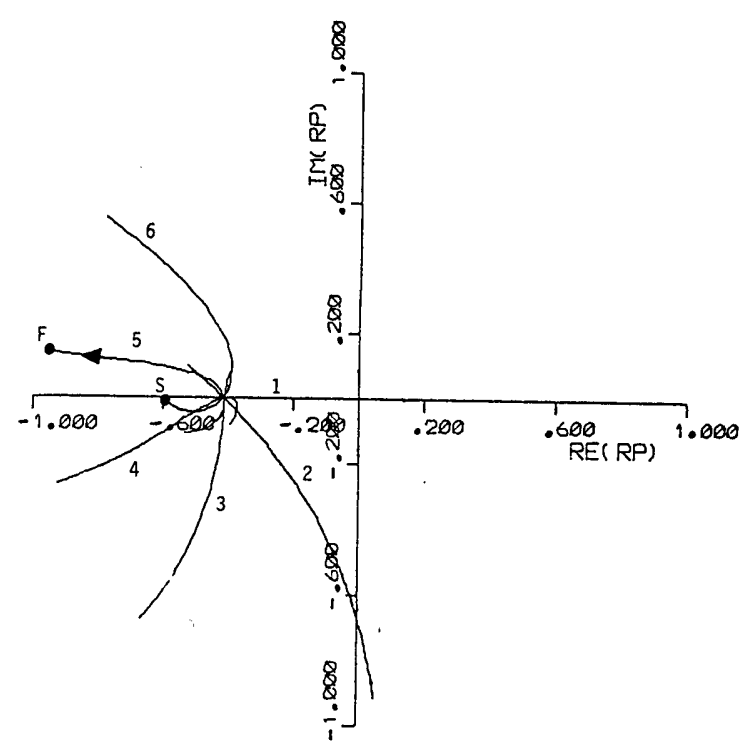

Fig. 15. Associated CTCs of $R_{p}$ for $d=(m / 5) D_{0}, m=0,1, \ldots, 5$. incidence is possible over a narrow interval of the reduced thickness range that includes $d=184.21 \mathrm{~nm}$. This may be expected from Fig. 11(a) as the CTC should continue to make a loop for thickness values close to and on both sides of that which corresponds to $m=17$. Notice, however, that the CTCs for $m=16$ and $m=18$ do not show loops, so that the thickness interval is less than $\sim 20 \mathrm{~nm}$.

It is reasonable to inquire whether this property of self-intersection of the CTC extends to the parallel polarization. Reference to Fig. 6 may lead us to believe that this is not so, because no CTC in that figure appears

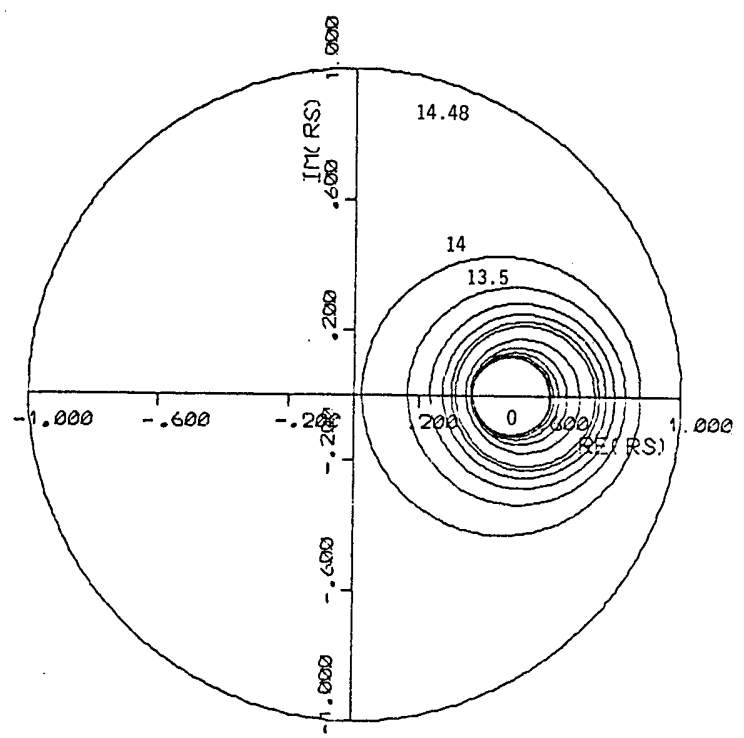

Fig. 16. CAICs of $R_{s}$ for partial internal reflection below the critical angle by the $\mathrm{Ge}^{-} \mathrm{ThF}_{4}$-air system at $\lambda=10.6 \mu \mathrm{m}$. The circles that lie between the $\phi=0$ and $\phi=13.5^{\circ}$ correspond to angles $\phi=2,4,6$, $8,10,11.65,12,12.5$, and $13^{\circ}$ in order of increasing radius. 


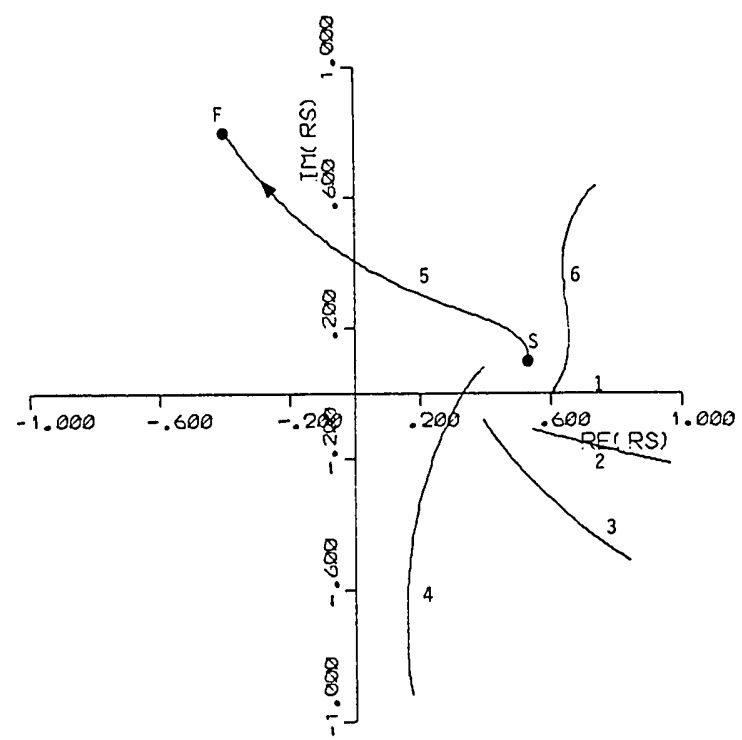

Fig. 17. CTCs of $R_{s}$ associated with Fig. 16 for $d=(m / 5) D_{0}, m=$

to form a loop. However, Fig. 19 shows a magnified view of a very small loop formed by the CTC of $R_{p}$ for $d=78.4 \mathrm{~nm}$. Interestingly, the loop disappears when $d$ is shifted from $d=78.4 \mathrm{~nm}$ by $<1 \mathrm{~nm}$ in either direction.

For film thicknesses well above the maximum period $\left(D_{90}=297.4 \mathrm{~nm}\right)$, the CTC can make one or more prominent loops. For example, Figs. 20(a), (b), and (c) show the CTCs of $R_{p}$ for (a) $d=0.5948 \mu \mathrm{m}$, (b) $d=$ $1.041 \mu \mathrm{m}$, and (c) $d=1.487 \mu \mathrm{m}$, respectively. Case (c) is one of two loops and four points of intersection, so that complex $R_{p}$ repeats itself for four distinct pairs of incidence angles.

Finally, Fig. 21 shows the corresponding CTCs of $R_{s}$ for the same film thicknesses that are assumed in Fig. 20.

\section{v. Summary}

The behavior of the complex reflection coefficients $R_{p}$ and $R_{s}$ of a film-substrate system for the $p$ and $s$ polarizations has been analyzed in more detail than heretofore attempted. Extensive results are presented graphically for the reflection of ( $\mathrm{He}-\mathrm{Ne}$ laser) light of wavelength $\lambda=0.6328 \mu \mathrm{m}$ by the air- $-\mathrm{SiO}_{2}-\mathrm{Si}$ system. Internal reflection of $\left(\mathrm{CO}_{2}\right.$-laser $) 10.6-\mu \mathrm{m}$ radiation by the $\mathrm{Ge}-\mathrm{ThF}_{4}$-air system is also considered.

Besides providing a better understanding of the reflection characteristics of polarized light by a filmsubstrate system, the analysis of this paper leads to some new results. In particular, it reveals the behavior of the pseudo-Brewster angle $\phi_{\mathrm{pB}}$ of a film-substrate system as a function of film thickness and indicates that the maximum possible value of the minimum parallel reflectance at $\phi_{\mathrm{pB}}$ is equal to the reflectance of the substrate at the Brewster angle of the ambient-film interface. We have also found that a film-substrate system can have the same complex reflection coefficient for the $p$ or $s$ polarization at two angles of incidence for certain ranges of film thickness. Useful applications of this interesting property remain to be seen.

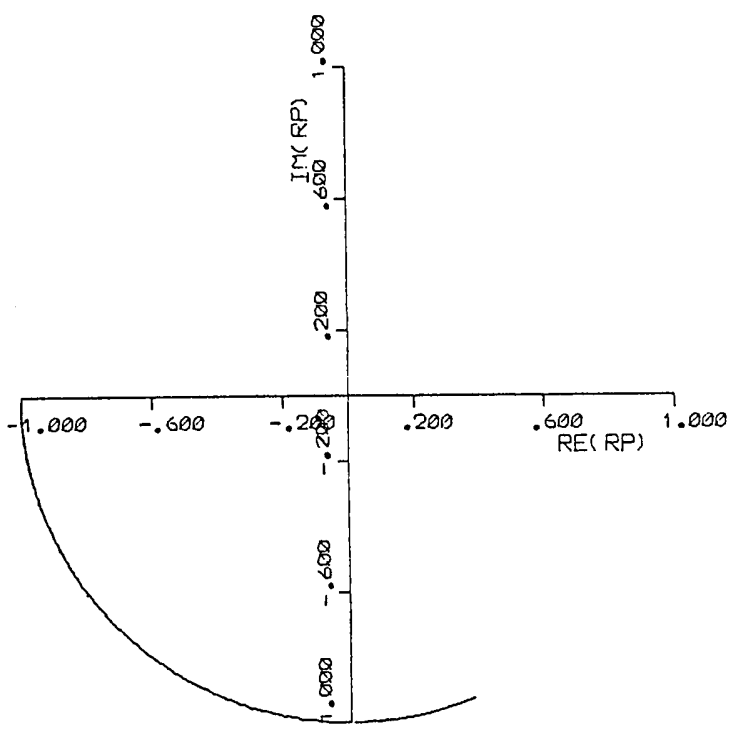

(a)

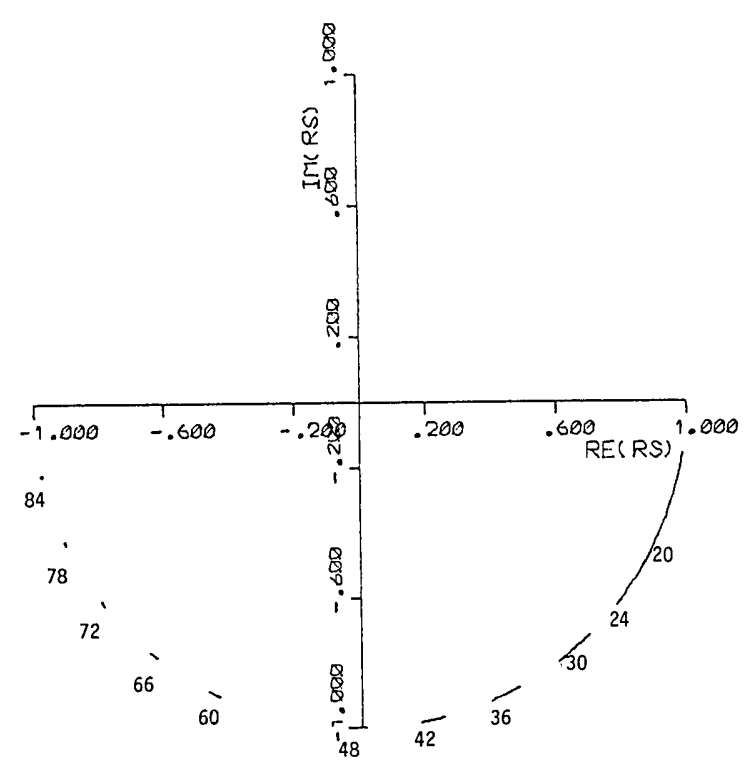

(b)

$0,1, \ldots, 5$.

Fig. 18. Family of overlapping (a) and nonoverlapping (b) circle arcs that represent the CAICs of $R_{p}$ and $R_{s}$, respectively, for total internal reflection above the critical angle by the $\mathrm{Ge}-\mathrm{ThF}_{4}$-air system at 10.6 $\mu \mathrm{m}$. 


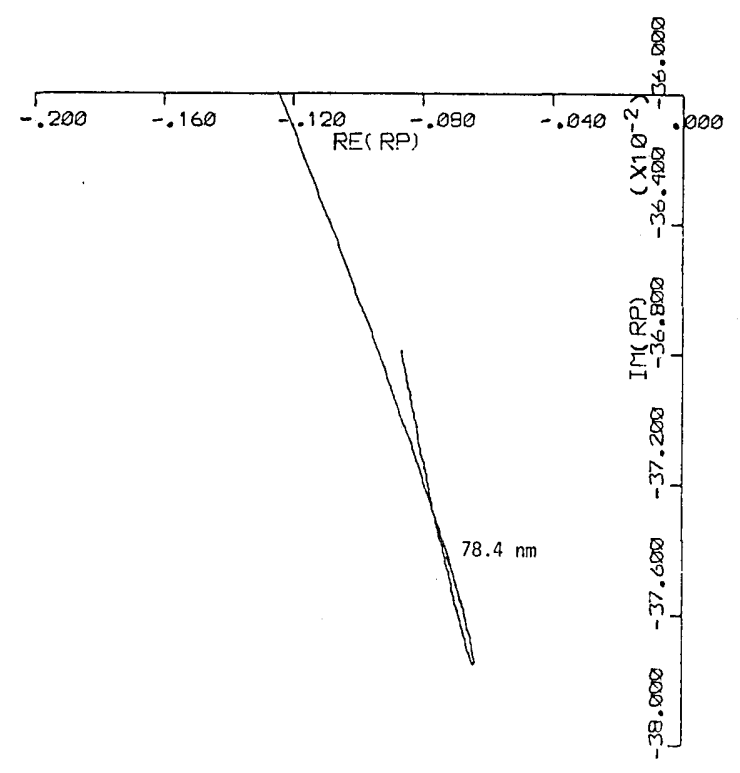

Fig. 19. CTC of $R_{p}$ for $\mathrm{SiO}_{2}$ film thickness $d=78.4 \mathrm{~nm}$ showing a small loop. The air-SiO ${ }_{2}-\mathrm{Si}$ system is assumed at $\lambda=0.6328 \mu \mathrm{m}$.

R. M. A. Azzam is pleased to acknowledge support of the National Science Foundation grant DMR-8018417. M. E. R. Khan is presently with the Intel Corp., Santa Clara, Calif. 95051.

Fig. 20. CTCs of $R_{p}$ for the air- $\mathrm{SiO}_{2}-\mathrm{Si}$ system for film thicknesses of (a) $0.5948 \mu \mathrm{m}$, (b) $1.041 \mu \mathrm{m}$, and (c) $1.487 \mu \mathrm{m}$. Each CTC intersects itself and hence shows one or more loops.

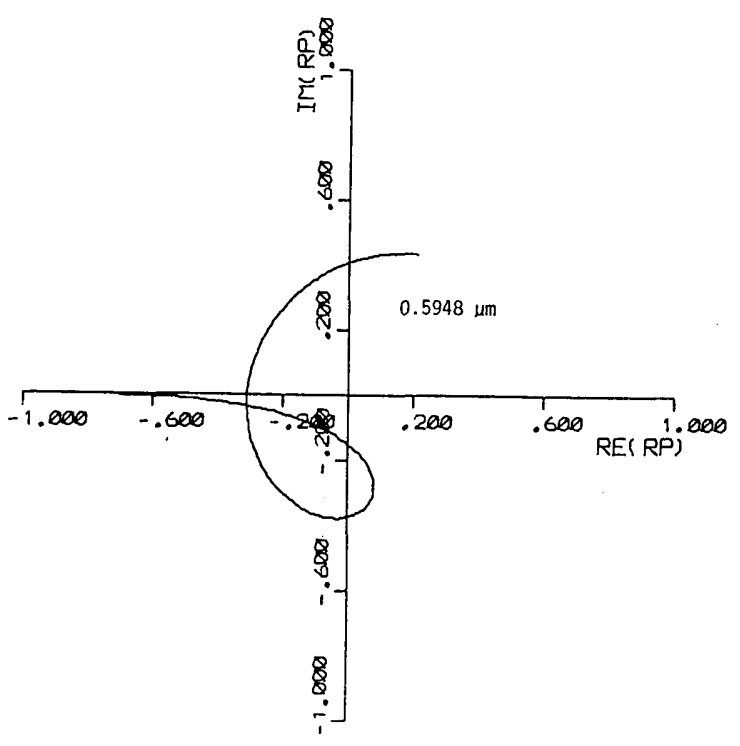

(a)

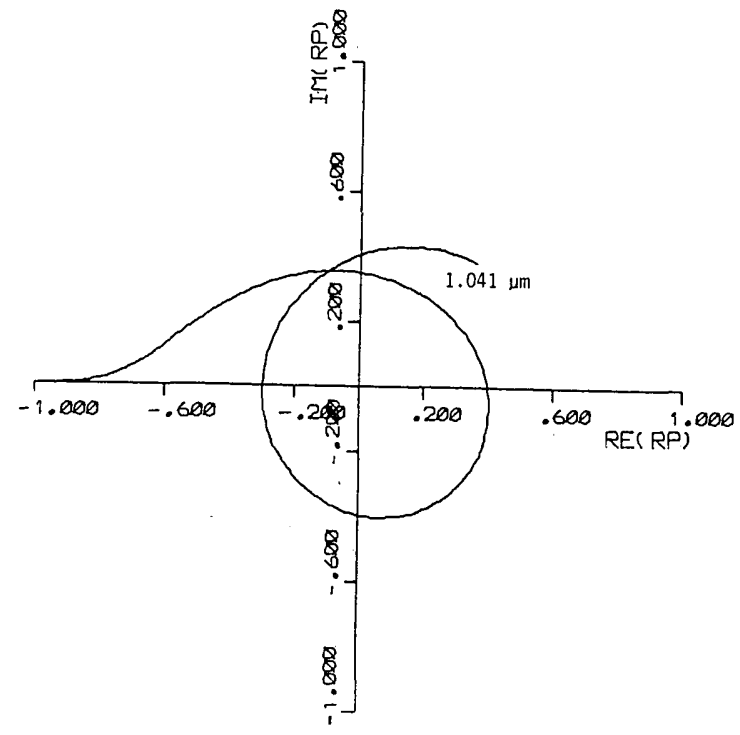

(b)

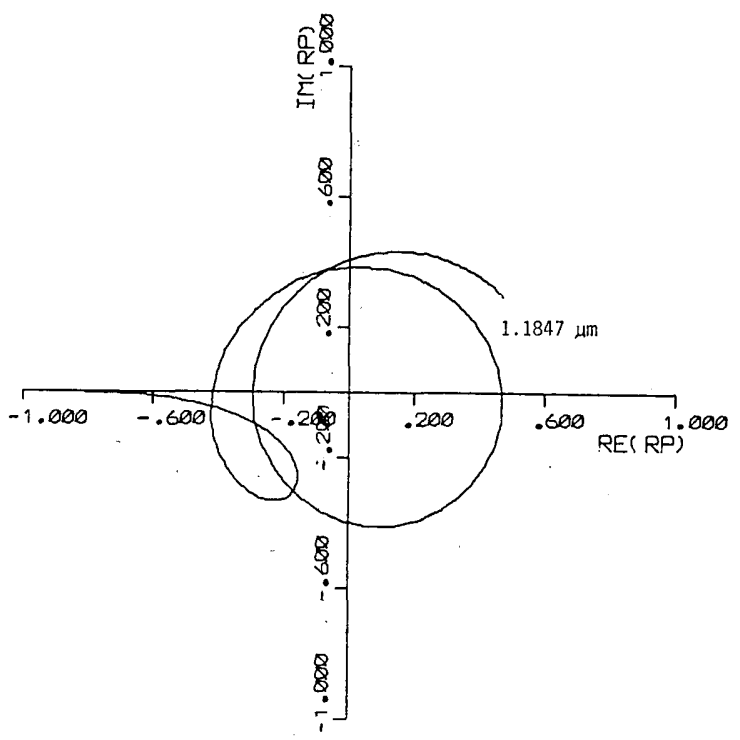

(c) 


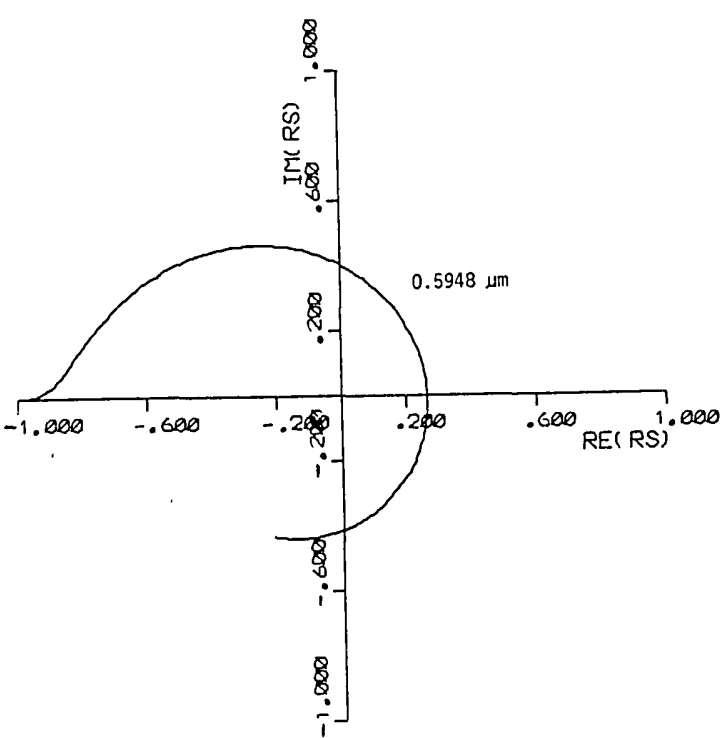

(a)

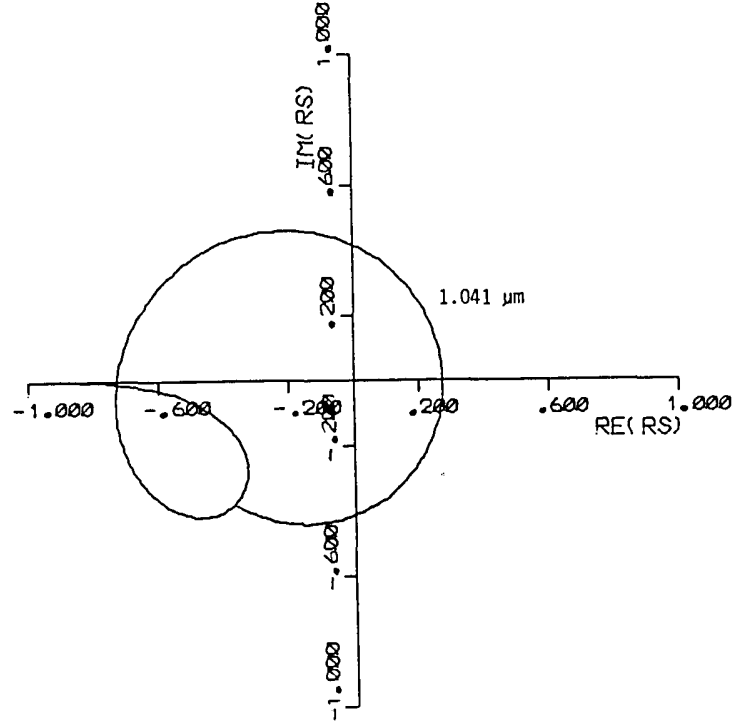

(b)

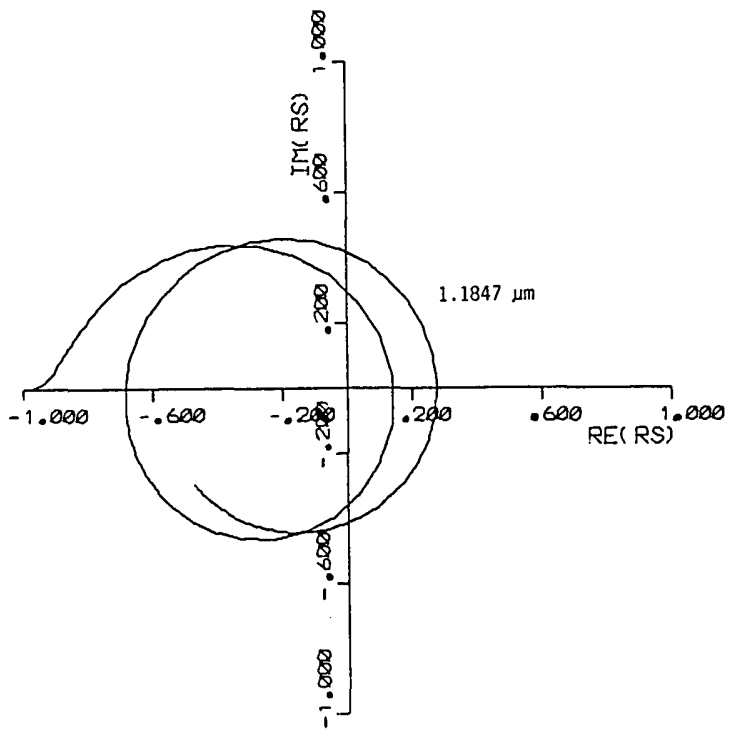

(c)

Fig. 21. Same as in Fig. 20 for $R_{s}$.

\section{References}

1. R. M. A. Azzam, A.-R. M. Zaghloul, and N. M. Bashara, J. Opt. Soc. Am. 65, 252 (1975).

2. A.-R. M. Zaghloul and R. M. A. Azzam, Appl. Opt. 21, 739 (1982).

3. G. Gergely, Ed., Ellipsometric Tables of the $\mathrm{Si}_{-} \mathrm{SiO}_{2} \mathrm{System}$ for Mercury and He-Ne Laser Spectral Lines (Akademiai Kaido, Budapest, 1971).

4. A. B. Winterbottom, in The Royal Norwegian Scientific Society Report 1 (F. Burns, Trondheim, 1955).

5. K. D. Naegele, J. Phys. Paris Colloq. C5 38, C5-225 (1977). This paper discusses some of the characteristics of the complex ratio $R_{\nu} / r_{02 \nu}$ of the reflection coefficient of the system $R_{\nu}$ to that of the ambient-substrate interface $r_{02 v}$.

6. M. D. Williams, Appl. Opt. 21, 747 (1982).

7. See, for example, R. M. A. Azzam and N. M. Bashara, Ellipsometry and Polarized Light (North-Holland, Amsterdam, 1977), Sec. 4.3.
8. See, for example, A. Kyrala, Applied Functions of a Complex Variable (Wiley-Interscience, New York, 1972), Sec. 8.4.

9. F. Abelès, J. Phys. Radium 11, 310 (1950).

10. The envelope is determined as follows: A given value of $\operatorname{Re}\left(R_{p}\right)$ $>-1$ is assumed, and $\phi$ is swept in search of a circle that intersects the line $\operatorname{Re}\left(R_{p}\right)=$ constant at a point that is most distant from the real axis [i.e., of maximum possible $\operatorname{Im}\left(R_{p}\right)$ for the given value of $\operatorname{Re}\left(R_{p}\right)$ ]. We have found that this procedure works better for this problem than an established method described by G. Zwikker, The Advanced Geometry of Plane Curves and their Applications (Dover, New York, 1963), Chap. 13.

11. W. L. Wolfe and G. J. Zissis, Eds., The Infrared Handbook (Office of Naval Research, Department of the Navy, Arlington, Va., 1978), p. 7-18.

12. M. E. Pedinoff, M. Braunstein, and O. M. Stafsudd, in Optical Polarimetry, R. M. A. Azzam and D. L. Coffeen, Eds., Proc. Soc. Photo-Opt. Instrum. Eng. 112, 74 (1977).

13. R. M. A. Azzam, Surf. Sci. 96, 67 (1980). 